\title{
Analysis of the Elements of Drag in Three-Dimensional Viscous and Inviscid Flows
}

\author{
R. M. Cummings* \\ California Polytechnic State University, San Luis Obispo, CA, U.S.A. \\ M. B. Giles ${ }^{\dagger} \quad$ G. N.Shrinivas ${ }^{\ddagger}$ \\ Oxford University Computing Laboratory, Oxford, UK.
}

\begin{abstract}
This paper examines the analytical, experimental, and computational aspects of tlie determination of the drag acting on an aircraft in flight, with or without powered engines, for subsonic/transonic flow. Using a momentum balance approach, the drag is represented by an integral over a cross-flow plane at an arbitrary distance behind the aircraft. Asymptotic evaluation of tlie integral shows tlie drag can be decomposed into three components corresponding to streamwise vorticity and variations in entropy and stagnation enthalpy. These are shown to be related to tlie established engineering concepts of induced drag, wave drag, profile drag and engine power and efficiency. This decomposition of the components of drag is useful in formulating techniques for accurately evaluating drag using computational fluid dynamics calculations or experimental data.
\end{abstract}

\section{Introduction}

The two most important aerodynamic quantities affecting an aircraft in flight are lift and drag. Nearly all aerodynamic analysis is an attempt to maximize the lift for a

of drag, or conversely to minimize tlie drag for of lift. Tlie analysis of these quantities for various aircraft configurations forms the basis of most aerodynamic research. Because of this, reliable methods to compute these forces from available experimental or are essential.

\footnotetext{
*Professor, Associate Fellow AIAA

${ }^{\dagger}$ Rolls-Royce Reader in CFD, Member AIAA

†DPhil student, email: nasng@comlab.ox.ac.uk

Copyright (C) 1996 by R.. M. Cummings, M. B. Giles and G. N. Shrinivas. Published by the American Institute of Aeronautics and Astronautics, Inc. with permission.
}

Traditionally, aerodynamic forces have been measured in wind tunnels using strain-gauge balances. This approach is very good for measuring the lift, but the drag of a typical aircraft at reasonable angles of incidence is often an order of magnitude less than the lift, and therefore more difficult to measure. In particular, the presence of the model sting or support nialces accurate drag measurement very difficult using this approach. This led to attempts to measure drag using techniques based on a control volume approach. The simplest application of this is to measure tlie momentum deficit parallel to the freestream within the wake of a model. The main drawback to this approach, however, was tlie need to perform the wake survey throughout the downstream flowfield, as well as various difficulties associated with tlie presence of tlie wind tunnel walls. An approach developed by Betz [1] modified the integral formulation to take into account the presence of the wind tunnel walls, and reduced the area of integration to tlie region directly behind the model. Unfortunately, Betz did not include terms which would account for tlie drag due to vortices, an important aspect of measuring the drag of a finite span wing. His approach was also found to have certain measurement difficulties as shown by Maull and Bearman [10]. In an attempt to correct some of tlie problems in Betz's approach, Maslcell [8] showed that an integral formulation could be obtained which would allow tlie measurement of both profile and vortex drag, both of which could be obtained from measurements in a reduced region behind the aircraft. Since that time various iinproveinents to model have been made for experimental measurements of drag; these include formulations due to $\mathrm{Wu}$ et al [14] and Brune and Bogataj [2].

As computational fluid dynamics (CFD) has matured over the years, it has become a goal of CFD researchers to be able to predict aerodynamic drag from numerical 
simulations. Early attempts at

were usually

met with frustration, as most approaches invol

grating the pressure

friction over the surface of

the body in order to calculate forces (the computational equivalent of force measurement in

Surface met

clue to the need to approximate the curved surface with flat facets, and tlie difficulty in accurately predicting friction. This has

to

attempt to experimental wake integral methods to CFD computations. Methods involving wake integratioii have been shown to accurate a.t predicting profile aiid vortex drag, as shown by van Dam aiid Nilcfetrat [12] and Jamus [3]. An equivalent lifting-line approach by Mathias et al [9] has also been sliowii to be able to accurately compute

The problem with tlie current approaches used to compute aerodynamic forces from CFD solutions is that various terms are usually neglected. These terms are known to be small far downstream of the aircraft, but in CFD calculations the wake becomes increasingly diffuse downstream because of iiuinerical smoothing, and so the integral methods need to he applied much closer to tlie aircraft. This carefully at tlie drag wake-survey methods aiid comes to an improved understanding of the importance of tlie various integrals and the terins that are often neglected. Tlie first approach is to take the cross-flow plane to be far downstream of the aircraft so that all flow components can he assumed to be approximately invariant in tlie freestream direction. This leads very simply to an integral form of the drag showing the different contributions due to streamwise vorticity aiid variatioiis in entropy aiid stagiiatioii enthalpy. Next, an analysis is performed for a plane which is much closer to the aircraft, and at which there is still significant flow variation in the freestream direction. Tlie same drag result is eventually obtained after careful analysis and appropriate asymptotic approximations. The purpose of this section is to relate the current analysis to the work of Betz [I],Maskell [8], Wu et al [14], Lock [7], van der Vooreii aiid Slooff [13], van Dam [12], Matliias et al [9] and others. In practice, experimental measurement planes are always in this near-field region aiid there lias been considerable discussion in the literature regarding the terms which should he included in tlie drag computation. It is shown in tlie analysis presented here that the terms clue to tlie poteiitial flow component of tlie velocity field cancel. A connection is also shown between the control volume foriiiulatioii and tlie classical lifting-line theory of induced drag, showing that the current analysis reduces to the classical analysis under certain limiting conditions. Tlie discuss the application of the theory to tion of drag from experiniental measurements or computational results.

\section{Control voluiiie foriiiulatioii}

The combined aerodynamic force can be written as an integral over the surface of an aircraft as,

$$
\boldsymbol{F}=\int_{\sigma}-p \boldsymbol{n}+\boldsymbol{\tau} \cdot \boldsymbol{n} d A
$$

where $p$ is the pressure, $\boldsymbol{n}$ is the surface

$\tau$ is tlie stress tensor. Using the integral form of the momentum equations the force can also be expressed as an integral over the surface of any control voluine enclosing the aircraft,

$$
\boldsymbol{F}=\int_{S}-p \boldsymbol{n}-\rho(\boldsymbol{u} \cdot \boldsymbol{n}) \boldsymbol{u}+\tau \cdot \boldsymbol{n} d A
$$

Conservation of mass for the same control volume requires that

$$
\int_{S} \rho(\boldsymbol{u} \cdot \boldsymbol{n}) d A=0
$$

aiid for any closed surface

$$
\int_{S} \boldsymbol{n} d A=0
$$

Therefore, if the far-field velocity relative to the aircraft is $U_{\infty}$ aligned with tlie $\mathrm{x}$-coordinate direction, equivalent form of tlie force integral is

$$
\boldsymbol{F}=\int_{S}-\left(p-p_{\infty}\right) \boldsymbol{n}-\rho(\boldsymbol{u} \cdot \boldsymbol{n})\left(\boldsymbol{u}-\boldsymbol{U}_{\infty}\right)+\tau \cdot \boldsymbol{n} d A
$$

If the control volume surface is sufficiently far from tlie aircraft, the viscous stress terms may be neglected aiid so tlie integral becomes

$$
\boldsymbol{F}=-\int_{S}\left(p-p_{\infty}\right) \boldsymbol{n}+\rho(\boldsymbol{u} \cdot \boldsymbol{n})\left(\boldsymbol{u}-\boldsymbol{U}_{\infty}\right) d A
$$

The control volume is to be a cube aligned with the $(x, y, z)$ coordinate axes aiid with the downstream face a fixed distance dowiistream of the aircraft. As tlie size of the cube increases, the contribution to tlie drag component of the integral from the other five faces teiids to zero. Therefore the final expression for tlie drag is

$$
D=-\iint p-p_{\infty}+\rho u\left(u-U_{\infty}\right) d y d z .
$$


is tlie common starting point for the development of methods of from experimental data. [6]. The lift is directly lly mounting the aircraft model on a sting aiid force using a.

drag is substantially smaller than tlie lift, its direct measurement is much to measurement error aiid so methods based on this control volume approach are often more accurate.

When using CFD methods, tlie aerodynamic forces on the aircraft can lie evaluated by direct numerical approximation of tlie integral in Equation (1), but even here there are benefits in using the drag integrals that result from tlie cross-flow plane analysis. These include elimination of spurious drag due to numerical smoothing; potentially faster steady-state convergence of the drag estimate in time-marching computations; avoidance of possible errors due to far-field boundary conditions; improved physical insight into the sources of drag for a particular aircraft configuration. These aspects are all discussed later in the relevant sections.

An additional integral which will be important for powered engines comes from the principle of energy conservation. If' thermal diffusion and work clue to viscous stresses are both negligible in tlie far-field, then energy conservation over the control volume surface $S$ gives

$$
E=\int_{S} \rho(\boldsymbol{u} \cdot \boldsymbol{n}) H d A
$$

where $I-I$ is the stagnation enthalpy aiid $E$ is the rate of energy input due to fuel combustion. Because of mass conservation, an equivalent form is

$$
E=\int_{S^{\prime}} \rho(\boldsymbol{u} \cdot \boldsymbol{n}) \Delta H d A
$$

where $A H \equiv H-H_{\infty}$. Taking the control volume to be cube as before, this leads to the integral

$$
E=\iint \rho u \Delta H d y d z
$$

evaluated on the downstream cross-flow plane.

\section{Far-field analysis}

downstream of the aircraft, the flow is approximately invariant in the $x$-direction. First, we consider a flow in is no streamwise vorticity. In this case, the flow velocity is purely in the $x$-direction and so $p(y, \mathrm{z})=p_{\infty}$ to $y$ and $z$ components of the momentum equations. Using the definitions of the stagnation ciithalpy a ropy,

$$
\begin{aligned}
& \frac{\mu}{(\gamma-1) \rho}+\frac{1}{2}\left(u^{2}+v^{2}+w^{2}\right. \\
& \log \left(\frac{p}{p_{\infty}}\right)-\gamma \log \left(\frac{\rho}{\rho_{\infty}}\right)
\end{aligned}
$$

with tlie freestream entropy defined to be zero, it follows that

$$
\begin{aligned}
\rho & =\rho_{\infty} \exp \left(-s / c_{p}\right) \\
71 & =\left(U_{\infty}^{2} \exp \left(s / c_{p}\right)+2\left(H-\mathrm{H} \quad \exp \left(s / c_{p}\right)\right)\right)^{\frac{1}{2}}
\end{aligned}
$$

These values can then be usecl to obtain the drag,

$$
D=-\iint \rho u\left(u-U_{\infty}\right) d y d z
$$

If thie entropy, $s$, and the perturbation in stagnation enthalpy, $A H \equiv H-H$, $\quad$ are both small then

$$
\begin{aligned}
u \approx\left(U_{\infty}^{2}+\right. & U_{\infty}^{2} \stackrel{\circ}{-}+2 \Delta H-2 H_{\infty} \frac{\circ}{} \\
& \left.+\frac{\Delta H}{U_{\infty}^{2}}-\frac{p_{\infty}}{\rho_{\infty} U_{\infty}^{2}} \frac{s}{R}\right)
\end{aligned}
$$

neglecting terms which are $O\left(s^{2}, s \Delta H,(A H)\right.$ ') and hence the drag is

$$
D \approx \iint p_{\infty} \frac{s}{R}-\rho_{\infty} \mathrm{AH} d y d z
$$

In inviscid flow without powered engines $\mathrm{AH}$ is zero aiid this reduces to the standard integral for transonic wave drag, first derived by Oswatitsch [11]. In viscous flow without powered engines $\mathrm{A} \mathrm{H}$ is usually still negligible. The increased entropy associated with tlie drag now conies from both the shocks and disspatioii in the boundary layer and wake, aiid so tlie drag integral is the combination of what is usually referred to as wave drag and profile drag. In the outflow from powered engines, A H is positive corresponding to tlie by the engine. The entropy will also be positive due to the inevitable thermodynamic cycle inefficiency aiid aerodynamic losses in the engine.

We now coiisider a flow with uniform entropy aiid stagnation enthalpy and streamwise vorticity $\zeta(y, z)$. The velocity field now has

$$
\boldsymbol{u}=(u(y, z), 0,0)
$$

$$
u=\left(U_{\infty}, v(y, z), w(y, z)\right)
$$


To leading order, the density is uniform and so the mass equation requires that

$$
\frac{\partial v}{\partial y}+\frac{\partial w}{\partial z}=0
$$

It is therefore possible to define $v$ and $w$ in terms of a cross-flow streamfunction $\psi$,

$$
v=\frac{\partial \psi}{\partial z}, \quad w=-\frac{\partial \psi}{\partial y}
$$

which must satisfy the streamfunction/vorticity equation

$$
\nabla^{2} \psi=-\zeta
$$

When the entropy and stagnation enthalpy are both uniform. the pressure is related to the flow speed, $q \equiv \sqrt{u^{2}+v^{2}+w^{2}}$, by

$$
\frac{p}{p_{\infty}}=\left(\frac{H_{\infty}-\frac{1}{2} q^{2}}{H_{\infty}-\frac{1}{2} U_{\infty}^{2}}\right)^{\frac{\gamma-1}{\gamma}}
$$

and hence

$$
\frac{d p}{d\left(q^{2}\right)}=-\frac{\gamma-1}{2 \gamma} \frac{p}{H_{\infty}-\frac{1}{2} q^{2}}=-\frac{1}{2} \rho .
$$

Since $q^{2}=U_{\infty}^{2}+v^{2}+w^{2}$, this gives

$$
\Delta p \approx-\frac{1}{2} \rho_{\infty}\left(v^{2}+w^{2}\right)
$$

and so the drag is

$$
D=\frac{1}{2} \rho_{\infty} \iint v^{2}+w^{2} \mathrm{dy} d z
$$

The simple physical interpretation of this equation is that thie moving aircraft is doing work on the surrounding air at rate $D U$, which must equal the rate at which it is leaving, in its wake, kinetic energy associated with the cross-flow.

It is possible to leave the integral in this form, but it is more convenient to express the velocity components in terms of the streamfunction and integrate by parts to obtain the following result, first obtained by Maskell [8],

$$
\begin{aligned}
D & =\frac{1}{2} \rho_{\infty} \iint\left(\frac{\partial \psi}{\partial y}\right)^{2}+\left(\frac{\partial \psi}{\partial z}\right)^{2} d y d z \\
& =-\frac{1}{2} \rho_{\infty} \iint \psi \frac{\partial^{2} \psi}{\partial y^{2}}+\psi \frac{\partial^{2} \psi}{\partial z^{2}} d y d z \\
& =\frac{1}{2} \rho_{\infty} \iint \psi \zeta d y d z .
\end{aligned}
$$

There are three attractive features to

The

first, is is 11011-zero in only a limited area of the cross-flow plane so the be performed over a This is particularly important for experimental purposes, allowing a reduction in the area survey required to determine the vorticity, from which the corresponding streamfunction is computed and then the integral is approximated. The second attraction is that tlie value of this integral is fairly insensitive to the streamwise location of the plane on which it is evaluated. Therefore, although it has been derived based on the assumption that the plane is in the far-field of the aircraft flow field, it can in fact be evaluated on a plane which is well within the near-field. The third feature is that it shows clearly the relationship between this component of drag and the shed vorticity associated with the lift on a. finite-span aircraft. This corresponds to the 'induced drag' of classical lifting-line theory; this relationship is further developed in a later section.

For a flow field variations in and stagnation enthalpy in addition to streamwise vorticity, the two analyses can be approximately combined by adding the respective drag components, neglecting higher order terms, to obtain

$$
D \approx D_{1}+D_{2}+D_{3},
$$

where

$$
\begin{aligned}
& D_{1}=p_{\infty} \iint \frac{s}{R} d y d z \\
& D_{2}=-\rho_{\infty} \iint \Delta H d y d z \\
& D_{3}=\frac{1}{2} \rho_{\infty} \iint \psi \zeta d y d z .
\end{aligned}
$$

This equation corresponds to Equation (10.28) in reference [13], if $D_{3}$ is kept in its cross-flow kinetic energy form, as in Equation (26) above.

In an experiment or a computation, each of the three integrals will be a weak function of the streamwise position of the plane on which they are evaluated. As explained in Section 2, while moving downstream $D_{2}$ will approach a constant value $-E / U_{\infty}$, where $\mathrm{E}$ is the rate of energy addition in the engines. $D_{3}$ will decay very slowly to zero as the streamwise vorticity diffuses until the vorticity shed by one wing cancels the vorticity of the opposite sign shed by the other wing. In a CFD computation, because of numerical smoothing and coarse grids in the far-field this will take place within the first, 100 aircraft, lengths; in reality it would take very much longer'. As $D_{3}$ decreases there is a corresponding increase in $D_{1}$ since the total drag remains a constant. 
In fact, tlie sum of the three components will be approxwell intr the near-field of tlie aircraft. This is fortunate becaus experimental measurements will usually have to be te ken in the near-field. Also, if a detailed breakdown of he sources of drag in a CFD calculation is required it is best to evaluate the three integrals in tlie near-field before numerical smoothing causes a shift from $D_{3}$ to $D_{1}$.

The lift can also be re ated, approximately, to the streamwise vorticity in the far-field. This result was first obtained for starting point is the flow by Maslcell [8]. The of tlie total force vector as an integral over he surface of a.cubic control volume enclosing the aircs ift, as in Equation (6),

$$
\boldsymbol{F}=-\int_{S}\left(p-p_{\infty}\right) \boldsymbol{n}+,(\boldsymbol{u} \cdot \boldsymbol{n})\left(\boldsymbol{u}-\boldsymbol{U}_{\infty}\right) d A
$$

Neglecting are quadratic in tlie perturbation velocities, the pressure perturbation on tlie side planes is related to tlie flow velocity perturbation by

$$
\Delta p \approx-\rho_{\infty} U_{\infty} \Delta u
$$

and hence the lift is

$$
L \approx \rho_{\infty} U_{\infty} \int_{S}\left(\Delta u n_{2}-w n_{x}\right) d \Lambda
$$

Using the following identity, in which $j$ is the unit vector in and $v$ is an arbitray vector field.

$$
\begin{aligned}
\nabla \times(y \boldsymbol{v}) & =(\nabla y) \times v+y(\nabla \times v) \\
& =j \times v+y(\nabla \times v)
\end{aligned}
$$

it follows that

$$
\begin{aligned}
L & \approx-\rho_{\infty} U_{\infty} \int_{S} \boldsymbol{n} \cdot(\boldsymbol{j} \times \Delta \boldsymbol{u}) d A \\
& =\rho_{\infty} U_{\infty} \int_{S}(y \boldsymbol{n} \cdot(\nabla \times \boldsymbol{u})-\boldsymbol{n} \cdot(\nabla \times(y \Delta \boldsymbol{u}))) d A
\end{aligned}
$$

For any vector field $v$ and any closed surface $S$,

$$
\int_{S} n \cdot(\nabla \times v) d A=0
$$

Also, the flow vorticity is noli-zero only on stream face of the cube. Therefore, result is

$$
L \approx \rho_{\infty} U_{\infty} \iint y \zeta d y d z
$$

with the integration being over just the dowiistreain cross-

\section{Near-field analysis}

In tlie near field in are significant variations in tlie $\mathrm{x}$-direction the velocity field can be expressed using a Clebsch decomposition as

$$
u=\nabla \phi+\nabla \times \psi
$$

where $\psi$ is now a vector function which satisfies the equation

$$
\nabla^{2} \psi=-\zeta
$$

with $\zeta$ being the vorticity vector. It is convenient to split $\psi$ into the streamwise part $\psi i$ and the remainder, so that

$$
\boldsymbol{u}=\nabla \phi+\nabla \times(\psi i)+u_{u} .
$$

The term $\boldsymbol{u}_{w}$ associated with the transverse vorticity is non-zero only in

Its dominant component is in the streamwise direction and so it corresponds to the velocity defect related to the variations in entropy and stagnation enthalpy, as discussed in the previous section. The link between transverse vorticity, entropy and stagnation enthalpy is also explicit in Crocco's theorem for steady flow,

$$
u \times \zeta=U H-T \nabla s .
$$

The drag due to this written as a function of stagnation enthalpy and entropy variations as before. Removing this term we now concentrate on the drag associated with tlie velocity field

$$
u=\nabla \phi+\nabla \times(\psi i)
$$

$$
\text { entropy and stagnation enthalpy. }
$$

Considering the pressure as a function of the flow speed, it was shown in the previous section that

$$
\frac{d p}{d\left(q^{2}\right)}=-\frac{1}{2} \rho
$$

Differentiating this again, gives

$$
\frac{d^{2} p}{d\left(q^{2}\right)^{2}}=-\frac{1}{2} \frac{d \rho}{d\left(q^{2}\right)}=-\frac{1}{2 c^{2}} \frac{d p}{d\left(q^{2}\right)}=\frac{\rho}{4 c^{2}} .
$$

The change in freestream speed is

$$
\Delta\left(q^{2}\right)=\left(U_{\infty}+\Delta u\right)^{2}+v^{2}+w^{2}-U_{\infty}^{2}
$$

so performing a second-order Taylor series expansion about freestream conditions gives

$$
\begin{aligned}
\Delta p & \approx-\frac{1}{2} \rho_{\infty} \Delta\left(q^{2}\right)+\frac{\rho_{\infty}}{8 c_{\infty}^{2}}\left(\Delta\left(q^{2}\right)\right)^{2} \\
& \approx-\frac{1}{2} \rho_{\infty}\left(v^{2}+w^{2}+2 U_{\infty} \Delta u+\left(1-M_{\infty}^{2}\right)(\Delta u)^{2}\right)
\end{aligned}
$$


To first order, the corresponding change in density is

$$
\Delta \rho \approx \frac{\Delta p}{c_{\infty}^{2}} \approx-\frac{\rho_{\infty} U_{\infty}}{c_{\infty}^{2}} \Delta u
$$

and so

$$
\begin{aligned}
\rho u \Delta u & \approx \rho_{\infty} U_{\infty} \Delta u+\rho_{\infty}(\Delta u)^{2}+\Delta \rho U_{\infty} \Delta u \\
& \approx \rho_{\infty} U_{\infty} \Delta u+\rho_{\infty}\left(1-M_{\infty}^{2}\right)(\Delta u)^{2}
\end{aligned}
$$

Putting these into the drag integral gives

$$
D=2 \quad \iint v^{2}+w^{2}-\left(1-M_{\infty}^{2}\right)(\Delta u)^{2} \mathrm{dydz} .
$$

This equation corresponds to Equation (10.20) of reference [13] when there is no variation in entropy or stagnation enthalpy. Following the approach of Maskell [8],

$$
\begin{gathered}
v=\frac{\partial \phi}{+}+\frac{\partial \psi}{\partial z}, \\
w=\begin{array}{l}
\partial d_{1} \\
\partial z
\end{array} \quad \partial y
\end{gathered}
$$

so integrating by parts gives

$$
\iint v^{2}+w^{2} d y d z=\iint \psi \zeta-\phi \sigma d y d z
$$

where

$$
\sigma \equiv \frac{\partial^{2} \phi}{\partial y^{2}}+\frac{\partial^{2} \phi}{\partial z^{2}}
$$

Combining these gives

$$
\begin{aligned}
D & =\frac{1}{2} \rho_{\infty} \iint \psi \zeta d y d z \\
& -\frac{1}{2} \rho_{\infty} \iint \phi \sigma d y d z \\
& -\frac{1}{2} \rho_{\infty} \iint\left(1-M_{\infty}^{2}\right)(\Delta u)^{2} d y d z .
\end{aligned}
$$

The first integral is exactly the same as appeared in the far-field analysis. The second integral appears in the analyses of Masltell [8] and Wu et al [14] but is usually ignored in practice on tlie grounds that $\sigma$ is small; this is essentially just the far-field argument used in the previous section. The third integral has been derived previously by Betz [1] for incompressible flow, and by Lock [7] and van der Vooren and Slooff [13] for coinpressible flow. Again it is usually argued that it is negligible.

In fact, to leading order the second and third integrals cancel. To prove this requires use of the mass equation which, to leading order, can be written as

$$
U_{\infty} \frac{\partial \rho}{\partial x}+\rho_{\infty} \nabla \cdot u=0
$$

From Equation (46),

$$
\frac{\partial \rho}{\partial x} \approx-\frac{\rho_{\infty} U_{\infty}}{c_{\infty}^{2}} \frac{\partial u}{\partial x}
$$

and so it lollows that

$$
\left(1-M_{\infty}^{2}\right) \frac{\partial^{2} \phi}{\partial x^{2}}+\frac{\partial^{2} \phi}{\partial y^{2}}+\frac{\partial^{2} \phi}{\partial z^{2}} \approx 0
$$

Hence, using integration by parts in both the $y$ and $z$ directions,

$$
\begin{aligned}
\frac{d z}{d x} \iint \phi \sigma d y d z & =\underset{d x}{d x} \iint \phi\left(\frac{\partial^{2} \phi}{\partial u^{2}}+\frac{\partial^{2} \phi}{\partial z^{2}}\right) d y d z \\
& =-\frac{d}{d x} \iint\left(\frac{\partial \phi}{\partial y}\right)^{2}+\left(\frac{\partial \phi}{\partial z}\right)^{2} d y d z \\
& =-2 \iint \frac{\partial^{2} \phi}{\partial y \partial x} \frac{\partial \phi}{\partial y}+\frac{\partial^{2} \phi}{\partial z \partial x} \frac{\partial \phi}{\partial z} d y d z \\
& =2 \iint \frac{\partial \phi}{\partial x} \frac{\partial^{2} \phi}{\partial y^{2}}+\frac{\partial \phi}{\partial x} \frac{\partial^{2} \phi}{\partial z^{2}} d y d z \\
& =-2 \iint 2\left(1-M_{\infty}^{2}\right) \frac{\partial^{2} \phi}{\partial x^{2}} \frac{\partial \phi}{\partial x} d y d z \\
& =-\frac{d}{d x} \iint\left(1-M_{\infty}^{2}\right)\left(\frac{\partial \phi}{\partial x}\right)^{2} d y d z
\end{aligned}
$$

Integrating this o.d.e. in tlie $\mathrm{z}$-direction with the boundary condition that both integrals tend to zero as $x+\infty$ gives the final result that

$$
\iint \phi \sigma \mathrm{dyd} z+\iint\left(1-M_{\infty}^{2}\right)(\Delta u)^{2} \mathrm{dydz}=0 .
$$

Thus, this analysis shows that it is correct to drop the potential flow term in Maskell's analysis, and keep only the terms due to the trailing axial vorticity and the entropy and stagnation enthalpy variations, as derived in the previous section. This result should not be surprising. In tlie absence of any shed vorticity or variation in entropy or stagnation enthalpy, all flow approach freestream conditions in tlie far-field and so there must be zero drag. As a consequence, the drag integral at any axial location in the near-field or the far-field must be identically zero.

\section{Connection to lifting-line theory}

In classical lifting line theory, the wing is assumed to have an extremely high aspect ratio and sheds a flat sheet of streamwise vorticity behind it. To satisfy Kelvin's circulation theorem, the strength of tlie 
shed vortex sheet $\gamma(y)$ is related to the circulation $\Gamma(y)$ around the airfoil sectioii,

$$
\gamma(y)=-\frac{d \Gamma}{d y}
$$

Using standard lifting-line theory, tlie drag is given by

$$
D=\rho_{\infty} U_{\infty} \int_{-b / 2}^{b / 2} \Gamma\left(y_{o}\right) \alpha_{i}\left(y_{o}\right) d y_{o}
$$

where $\alpha_{i}\left(y_{0}\right)$ is

at $y_{0}$ due to

tlie shed vorticity along the span. Using tlie Biot-Savart Law for the senii-infinite vortex lines trailing behind tlie wing, the induced downwash is given by

$$
\alpha_{i}\left(y_{o}\right)=-\frac{1}{4 \pi U_{\infty}} \int_{-b / 2}^{b / 2} \frac{\gamma(y)}{y_{0}-y} d y .
$$

'The integrand is singular at $\mathrm{y}=y_{0}$; only the principal part is retained. Coinbining these two equations gives

$$
\begin{aligned}
D & =-\frac{\rho_{\infty}}{4 \pi} \int_{=k, / 2}^{b / 2} \int_{=k / 2}^{b / 2} \frac{\Gamma\left(y_{o}\right) \gamma(y)}{y_{o}-y} d y d y_{o} \\
& =\int_{-b / 2}^{b / 2} \gamma(y) f(y) d y
\end{aligned}
$$

where

$$
f(y)=-\frac{\rho_{\infty}}{4 \pi} \int_{-b / 2}^{b / 2} \Gamma\left(y_{o}\right)-y d y_{0}
$$

We now consider tlie drag given by the formula

$$
D=\frac{1}{2} \rho_{\infty} \iint \psi \zeta d y d z
$$

where tlie streamfunction $\psi$ satisfies Poisson's equation

$$
\nabla^{2} \psi=-\zeta
$$

The general solution to this equation, subject to the boundary condition that $\nabla \psi \rightarrow 0$ as $y^{2}+z^{2} \rightarrow \infty$, is

$\psi(y, z)=-\frac{1}{4 \pi} \iint \zeta\left(y_{0}, z_{o}\right) \log \left(\left(y-y_{0}\right)^{2}+\left(z-z_{o}\right)^{2}\right) d y_{0} d z_{0}$

When $\zeta$ is a distributed line source of strength $\gamma(y)$ along $z_{0}=0$, thie integral becomes

$\psi(y, z)=-4 \pi \int_{-b / 2}^{u, / 2} \gamma\left(y_{0}\right) \log \left(\left(y-y_{0}\right)^{2}+z^{2}\right) d y_{0}$.

Integrating by parts, and using the fact that tlie circulation goes to zero at each wingtip, gives

$$
\psi(y, z)=-\frac{1}{2 \pi} \int_{-b / 2}^{b / 2} \frac{\Gamma\left(y_{0}\right)\left(y_{o}-y\right)}{\left(y-y_{0}\right)^{2}+z^{2}} d y_{o}
$$

Taking tlie limit $z \rightarrow 0$, this gives the induced drag as

$$
D=\int_{-b / 2}^{b / 2} \gamma(y) f(y) d y
$$

where, as before,

$$
f(y)=-\frac{\rho_{\infty}}{4 \pi} \int_{-b / 2}^{b / 2} \frac{\Gamma\left(y_{o}\right)}{y_{o}-y} d y_{o} .
$$

per unit span is $\rho_{\infty} U_{\infty} \Gamma(y)$, and so

$$
L=\rho_{\infty} U_{\infty} \int_{-b / 2}^{b / 2} \Gamma(y) d y .
$$

Integrating by parts once gives

$$
L=\rho_{\infty} U_{\infty} \int_{=k / 2}^{b / 2} y \gamma(y) d y .
$$

This corresponds precisely to the lift integral derived earlier for a general distribution of streamwise vorticity at the cross-flow plane, Equation (36), in the limit in which the vorticity is concentrated into a vortex sheet.

Thus, in the case of a planar vortex sheet tlie streainfunctioii-vorticity lift and drag integrals give tlie same result as classical lifting-line theory. The advantage of the streainfunctioii-vorticity approach over the lifting-line theory is that much more general ability to handle noli-planar trailing ity, due to winglets, pylons, complex boundary layer separations, etc. The advantage of the lifting-line theory is its extreme simplicity for simple applications and its ability to directly prove that an elliptic lift distributhe induced drag of a wing of fixed span [9],

\section{Experiineiital iiieasureineiits}

Experiineiital wake surveys have traditionally employed four-hole or five-hole probes from which one obtains the static and stagnation pressures, as well as all three velocity components.

are also being considered as a way to obtain these properties [2]. The generally accepted method for computed induced drag based on such wake surveys is to compute tlie streamwise vorticity by differentiating the velocity field using

$$
<=\frac{\partial w}{\partial y} \quad \frac{\partial v}{\partial z}-
$$

This method of computing tlie streaiiiwise vorticity can lead to errors in the prediction of the induced drag 
due to the differentiation of the discrete velocity measurements. One solution to this problem involves curvefitting the in order $t$, obtain more accurate derivatives [2]. An alternative approach is to relate the crossflow vorticity to circulation, and thus reintegration.

For region $C$ in a. cross-flow area. integral of the streamwise vorticity is equal to the circulation boundary of $C$,

$$
\int_{C} \zeta d y d z=\oint_{\partial C} v d y+w d z
$$

Therefore, given the values of the cross-flow velocity components $v_{j, k}$ and $w_{j, k}$ a.t a uniform grid of measure-

$$
\text { a. cross-plane (as }
$$

Figure I) the streamwise component of vorticity $\zeta$ measurement 'cell' can lie approximated by

$$
\zeta_{j+\frac{1}{2}, k+\frac{1}{2}}=\frac{\Gamma_{j+\frac{1}{2}, k+\frac{1}{2}}}{\Delta y \Delta z}
$$

with the circulation $\Gamma_{j+\frac{1}{2}, k+\frac{1}{2}}$ defined by

$$
\begin{aligned}
\Gamma_{j+\frac{1}{2}, k+\frac{1}{2}}= & \frac{1}{2}\left(v_{j, k}+v_{j+1, k}\right) \Delta y \\
& +\frac{1}{2}\left(w_{j+1, k}+w_{j+1, k+1}\right) \Delta z \\
& -\frac{1}{2}\left(v_{j+1, k+1}+v_{j, k+1}\right) \Delta y \\
& -\frac{1}{2}\left(w_{j, k+1}+w_{j, k}\right) \Delta z .
\end{aligned}
$$

For an arbitrary distribution of vorticity, the streamfunction $\psi(y, z)$ is

$\psi(y, z)=-\frac{1}{4 \pi} \iint \zeta\left(y_{o}, z_{0}\right) \log \left(\left(y-y_{0}\right)^{2}+\left(z-z_{o}\right)^{2}\right) d y_{o} d z_{o}$

Approximating the integral using the formulation first described by Lamb [5] gives

$$
\begin{aligned}
\psi_{j, k}=-\frac{1}{4 \pi} \sum_{j_{o}, k_{o}} \Delta y \Delta z \zeta_{j_{o}+\frac{1}{2}, k_{o}+\frac{1}{2}} \log & \left(\left(j_{o}+\frac{1}{2}-j\right)^{2} \Delta y^{2}\right. \\
& \left.+\left(k_{o}+\frac{1}{2}-k\right)^{2} \Delta z^{2}\right) \\
=-\frac{1}{4 \pi} \sum_{j_{o}, k_{o}} \Gamma_{j_{o}+\frac{1}{2}, k_{o}+\frac{1}{2}} \log ( & \left(j_{o}+\frac{1}{2}-j\right)^{2} \Delta y^{2} \\
+ & \left.\left(k_{o}+\frac{1}{2}-k\right)^{2} \Delta z^{2}\right)
\end{aligned}
$$

Finally, the drag integral can be approximated by summing over each cell to give the induced drag as

$$
D=\frac{1}{2} \rho_{\infty} \sum_{j, k} \frac{1}{4}\left(\psi_{j, k}+\psi_{j+1, k}+\psi_{j, k+1}+\psi_{j+1, k+1}\right) \Gamma_{j+\frac{1}{2}, k+\frac{1}{2}} \text {. }
$$

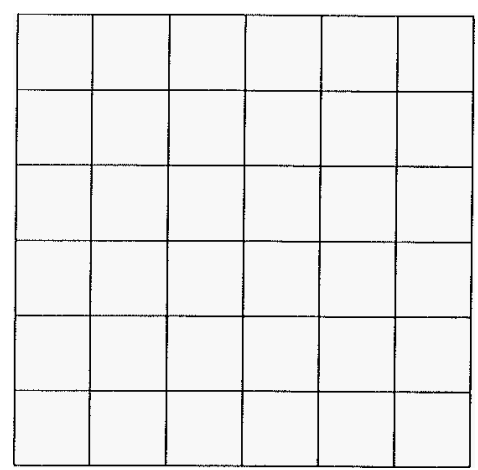

a) Cartesian

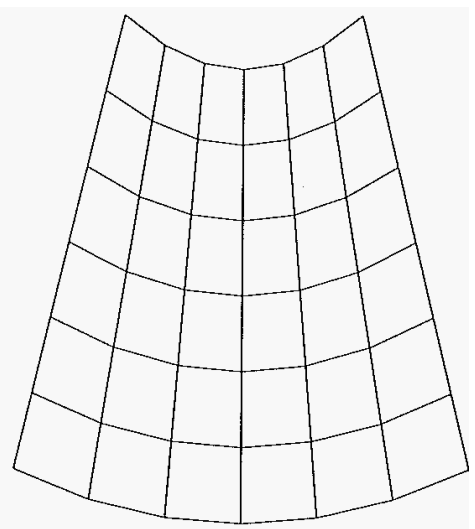

b) structured

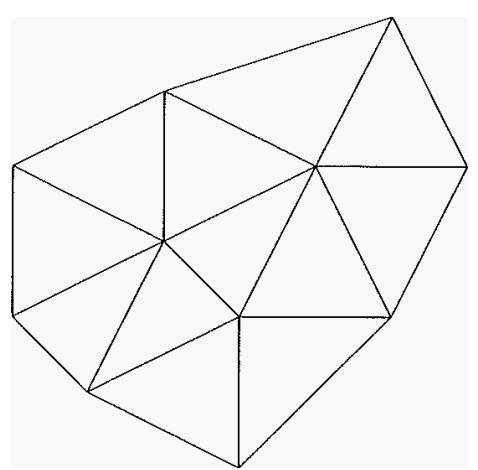

c) unstructured

Figure 1: Cross-flow grids for the evaluation of drag integrals 
of poivered

any significant

level of surface heat transfer, there is negligible variation in stagnation enthalpy. Therefore, tlie be deduced directly from tlie stagnation pressure aiid the corresponding drag integral is easily approximated.

If there are powered engines, then stagnation enthalpy (or stagnation temperature) measurements may need to be made. Alternatively, if $\Delta H$ cari be assumed to be small, tlie integral corresponding to the stagnation enthalpy related to tlie engines' fuel consumption; by energy conservation tlie net outflow of energy from must match the volume surrounding tlie aircraft release clue to fuel combustion.

\section{CFD computations}

For CFD calculations using unstructured grids, there is no crossflow plane in tlie computational grid, aiid so the approach for the evaluation of the crossflow drag integrals is to adopt techniques from flow visualisation. A cross-flow 'cutting be defined to be orthogonal to the freestream flow and at a fixed of the aircraft. Tlie grid nodes on this cutting are defined by the intersection of tlie tlie edges of the 3D grid and all flow also be defined at the new grid nodes by along tlie cut edges. Tlie nodes of the cutting planes are on of tlie cutting plane to

cut cells. Tlie full details for uiistructured grids composed of tetrahedra, prisms, hexahedra are a paper by a.mple of the [4]. grid is

Figure 1.

Oiice the triangular cutting-plane grid has structed, the evaluation of the drag integral is quite straightforward. Tlie circulation around a. triaiigular cell is

$$
\Gamma_{\alpha}=\sum_{\text {edges }}(\bar{v} \Delta y+\bar{w} \Delta z),
$$

where $\bar{v}$ and $\bar{w}$ are tlie average velocity an edge, aiid Ay aiid Az are tlie changes in y and $z$ aloiig the edge (going around the cell rection as viewed from $n:=m$ ).

$$
\begin{gathered}
j \text { is given by } \\
-\sum \Gamma_{\beta} \log \left(\left(y_{j}-y_{\beta}\right)^{2}+\left(z_{j}-z_{\beta}\right)^{2}\right),
\end{gathered}
$$

where $y_{\beta}, \approx \beta$ are of cell. Tlie induced drag integral is summation over all of the triangular cells,

$$
D=\frac{1}{2} \rho_{\infty} \sum_{\alpha} \bar{\psi}_{\alpha} \Gamma_{\alpha},
$$

where $\bar{\psi}_{\alpha}$ is the average of

values at tlie three corner nodes.

Two refinements to tlie above formulation reduce the computational cost of evaluatiiig tlie drag. The first addresses the problem that each streamfunction value requires a loop over all of the cells in the cross-flow plane. Therefore the total computational cost is proportional to the square of tlie number of cells, which can lie large for very fine grids. However, in a few cells have significant levels of circulation, aiid it is oiily these cells which are needed for an accurate drag evaluation. Substituting Equation (81)into Equation (82) gives

$$
D=\frac{1}{2} \rho_{\infty} \sum_{\alpha, \beta} \Gamma_{\alpha} \Gamma_{\beta} D_{\alpha \beta}
$$

where

$$
D_{\alpha \beta}=\frac{1}{2 \pi} \sum \log \left(\left(y_{j}-y_{\beta}\right)^{2}+\left(z_{j}-z_{\beta}\right)^{2}\right)
$$

with the $j$ summation being over the 3 nodes at tlie corners of cell $a$. Tlie drag summation, Equation (83), can be restricted to those values of $\alpha$ aiid $\beta$ for which tlie magnitudes of $\Gamma_{\alpha}$ and $\Gamma_{\beta}$ exceed

old. Setting tlie threshold to be $0.1 \%$ of the maximum circulation cell leads to a. negligible error in the drag summation but can give a huge reduction in tlie computational cost.

The

is for the common case in which tlie CFD computation is performed for one half of a.flow which is symmetric about $y=0$. Rather than constructing tlie other half of the flow field aiid then applying tlie above procedure, it is simpler to account for the image vorticity

tlie streamfunction as

$$
\begin{aligned}
\psi_{j}=-\frac{1}{4 \pi} \sum_{\beta} \Gamma_{\beta} & \left\{\log \left(\left(y_{j}-y_{\beta}\right)^{2}+\left(z_{j}-z_{\beta}\right)^{2}\right)\right. \\
& \left.-\log \left(\left(y_{j}+y_{\beta}\right)^{2}+\left(z_{j}-z_{\beta}\right)^{2}\right)\right\} .
\end{aligned}
$$

For calculations on single-block aiid multi-block structured grids, it is unlikely that there exists a suitable grid coordinate plane which is at a uniform streainwise of tlie aircraft. One option is to use the same 'cutting plane' approacli just presented, creating an unstructured triangular grid on the crossflow data interpolated aloiig tlie cut edges of the structured grid. 
is possible when there are $i$ and $i+1$ which are approximately cross-flow planes. For $3 \mathrm{D}$ volume $D$, the of vorticity is related to a. surface integral of the velocity,

$$
\int_{D} \zeta d V=\int_{\partial D} u \times d S
$$

Therefore, using the 3D cell lying between coordinate planes $i$ and $i+1, j$ and $j+1$, aiid $k$ and $k+1$, a. value foi: the streamwise vorticity is given by

$$
\zeta_{j+\frac{1}{2}, k+\frac{1}{2}}=\frac{1}{V} \sum_{\text {laces }} i \cdot(\bar{u} \times S)
$$

where $V$ is tlie volume of the cell, $\bar{u}$ is the average velocity on the face aiid $S$ is tlie face area vector. This vorticity is a.nominal cross-flow plane on which tlie grid coordinates are

$$
y_{j, k}=\frac{1}{2}\left(y_{i, j, k}+y_{i+1, j, k}\right), \quad z_{j, k}=\frac{1}{2}\left(z_{i, j, k}+z_{i+1, j, k}\right)
$$

This noli-orthogonal structured grid is also illustrated in Figure 1. Tlie streamfunction $\psi_{j, k}$ is obtained from

$$
\begin{aligned}
\psi_{j, k}=-\frac{1}{4 \pi} \sum_{j_{o}, k_{o}} \Gamma_{j_{o}+\frac{1}{2}, k_{o}+\frac{1}{2}} \log \left(\left(y_{j, k}-y_{j_{o}+\frac{1}{2}, k_{o}+\frac{1}{2}}\right)^{2}\right. \\
\left.+\left(z_{j, k}-z_{j_{o}+\frac{1}{2}, k_{o}+\frac{1}{2}}\right)^{2}\right)(
\end{aligned}
$$

where the circulation of the cell is defined as

$$
\Gamma_{j_{o}+\frac{1}{2}, k_{o}+\frac{1}{2}}=A_{j_{0}+\frac{1}{2}, k_{o}+\frac{1}{2}} \zeta_{j_{0}+\frac{1}{2}, k_{o}+\frac{1}{2}}
$$

tlie coordinates at the centre of the cell are

$$
\begin{aligned}
y_{j+\frac{1}{2}, k+\frac{1}{2}} & =\frac{1}{4}\left(y_{j, k}+y_{j+1, k}+y_{j, k+1}+y_{j+1, k+1}\right), \\
z_{j+\frac{1}{2}, k+\frac{1}{2}} & =\frac{1}{4}\left(z_{j, k}+z_{j+1, k}+z_{j, k+1}+z_{j+1, k+1}\right),
\end{aligned}
$$

aiid $A$ is the cell area..

Finally, the induced drag integral is approximated by

$$
D=\frac{1}{2} \rho_{\infty} \sum_{j, k} \frac{1}{4}\left(\psi_{j, k}+\psi_{j+1, k}+\psi_{j, k+1}+\psi_{j+1, k+1}\right) \Gamma_{j+\frac{1}{2}, k+\frac{1}{2}} .
$$

Tlie same two refinements which were described for tlie unstructured grid structured grid analysis.

The next issue is the interpretation of the values obtained from tlie drag integrals. Using CFD methods, it is possible to directly evaluate tlie aerodynamic force on tlie aircraft using a numerical approximation of the surface integral of Equation (1).

CFD methods are conservative, so if the surface force integration is performed in a manner consistent with the (IFD discretisation of the cells with surface faces, then it is possible to sum over a very large number of computational cells surrounding the aircraft and deduce numerical surface force integral is exactly equal to that which would lie obtained by a numerical force/momentum

to Equa-

tion (5) applied on the enclosing

In

the far-field, numerical smoothing

the real viscous effects, are very small. Therefore, the far-field asymptotic

iiierical force integral on the showing that the nu-

equated to the drag integrals on the cross-flow plane.

This raises the question of what is to be gained from evaluating the drag using tlie cross-flow plane integrals rather than the direct surface integration. There are in fact four benefits in using the cross-flow integrals:

1. In subsonic Euler analysis shows

far-field drag one due to tlie streaiiiwise vorticity arising as a consequence of the spanwise lift distribution is physically meaningful and should have very nearly tlie correct physical Euler calculations give relatively accurate lift predictions. Tlie second contribution due to entropy variations is almost entirely spurious. Physically there should be a.slight level of entropy rise clue to some early diffusion of the shed vorticity, but in the CFD computation almost all of the entropy will be due to numerical smoothing in regions with high flow gradients aiid inadequate grid resolution, especially near tlie leading edge of tlie wing. As a consequence, a more accurate prediction of the real aircraft drag is obtained by entirely neglecting the entropy drag integral, keeping only the induced drag streamwise vorticity integral.

For transonic Euler calculations with shocks, and for Navier-Stokes calculatioiis with entropy generation in tlie boundary layer, it is much harder to distinguish between physically correct entropy generation and spurious numerical generation, so it may not be possible to apply such a correction.

2. If the boundaries are not sufficiently far from the aircraft, or if tlie are not sufficiently accurate (e.g. do not incorporate tlie farfield correction due to the lift on the aircraft) then there may be a very siiiall error in the effective freestream flow angle. This will produce only a. small error in lift but can produce a more significant error in drag since tlie effective rotation of the lift vector means that the lift will contribute an apparent drag component of magnitude $L \Delta \alpha$. 'This problem is totally avoided by use of 
of the drag. 'The streamwise component of vorticity is only very slightly altered by a.slight error in the freestream flow angle, so the relative drag error will be extremely small.

When there are no powered engines, or when the stagnation enthalpy variation is sufficiently mixed out that it can be equated to the energy input to the engines, the drag depends solely on the entropy variations and the streamwise vorticity. These quantities change very little during tlie final stages of time-marching convergence to the steady-state solution. Therefore, the drag integral based on the downstream cross-flow plane will converge to the final steady-state value quicker than the force integral over the surface of the aircraft. In practical CFD computations, this should allow fewer computational iterations to be required to obtain a given level of convergence of both tlie lift and drag.

4. Even if there were no quantitative advantages in expressing the drag in terms of the cross-flow integrals, there is still a. major qualitative benefit. Engineering analysis is simply one step in the process of engineering design, creating a better product. From this design viewpoint, it is important to not only know the value of overall drag but to also understand the causes of that drag so that design decisions can be made to hopefully reduce it. For example, a high level of induced drag for a given span and overall lift would suggest a poor spanwise lift distribution which might be improved by changing the spanwise variation in the angle of attack or re-cambering certain parts of the wing. Alternatively, a large entropy drag might be clue to either poor wave drag due to shocks or poor profile drag clue to a boundary layer separation. This would therefore suggest areas of further study of the detailed CFD computation.

\section{Evaluation of drag coinputations}

Two tests cases are used to validate the numerical discretisation and programming implementation of the induced drag integral. The first is the wake behind an elliptically loaded planar wing. Using a unit semi-span, tlie spanwise lift distribution is taken to be $\Gamma(\theta)=\sin \theta$, where the spanwise coordinate is $\mathrm{y}=\cos \mathbf{0}$.

flow velocity field in is then given by

$$
\begin{aligned}
& v(y, z)=-\frac{1}{2 \pi} \int_{0}^{\pi} \frac{\omega}{(y-\cos \theta)^{2}+z^{2}} \cos \theta d \theta \\
& w(y, z)=\frac{1}{2 \pi} \int_{0}^{\pi} \frac{y-\cos \theta}{(\mathrm{y}-\cos \theta)^{2}+z^{2}} \cos \theta d \theta
\end{aligned}
$$

and the exact value for the drag is $\pi / 8$, assuming unit freestream density.

Using a Cartesian grid of size $20 \times 40$ for the regioii $0<y<2,-1<z<1$, with clustering to accurately capture the vortex sheet and the large velocity gradients around the wing tip (as shown in Figure 2), the error from tlie numerical induced drag integral is only $1.1 \%$. With a uniform Cartesian grid of the same size over the same region, the error increases to $15 \%$ showing the effect of the decreased wake resolution.

The second case is the wake behind an engine whose exhaust is not aligned with the freestream. Using polar coordinates, $\mathrm{y}=r \cos \mathbf{0}, z=r \sin 0$ and assuming a unit radius for the engine, the cross-flow velocity field is

$$
\begin{aligned}
& v= \begin{cases}0, & r<1 \\
-\frac{\sin 2 \theta}{r^{2}}, & r>1\end{cases} \\
& w= \begin{cases}-1, & r<1 \\
\frac{\cos 2 \theta}{r^{2}}, & r>1\end{cases}
\end{aligned}
$$

By integrating the cross-flow kinetic energy, the exact value for the drag is found to be $\pi$, again assuming a unit freestream density.

Using a polar grid of size $20 \times 40$ for $0 \leq r \leq 2$, with clustering to accurately capture the vortex sheet at $r=1$, (as shown in Figure 3), the error from the numerical induced drag integral is only $1.4 \%$. With a uniform Cartesian grid of the same size over the region $0 \leq \mathrm{y} \leq 1.5,-1.5 \leq z \leq 1.5$, the error increases to $4.4 \%$, again because of the effective smoothing of the velocity discontinuity across the vortex sheet.

The drag calculation methods were next tested on a case which had been both experimentally and computationally predicted. A simple rectangular wing model which used an untwisted NACA 0016 airfoil section was tested by Brune and Bogataj [2]. Tlie wing had an aspect ratio of six with rounded wing tip fairings, and was tested at $M=0.18, \alpha=8.22^{\circ}$ and $R e,=1.27 \times 10^{6}$. The model had boundary layer trip strips to ensure that the flow was turbulent over the majority of the wing surface. Table 1 presents averaged lift and drag coefficients obtained from both wake surveys and balance measurements. $C_{d i}^{\prime}$ is the induced drag computed from the streamwise vorticity in the wake survey, and $C_{d p}$ is the profile drag computed from the total pressure measured in the wake survey. Table 1 also shows lift and drag estimates calculated by Mathias et al using integration and an equivalent lifting line 

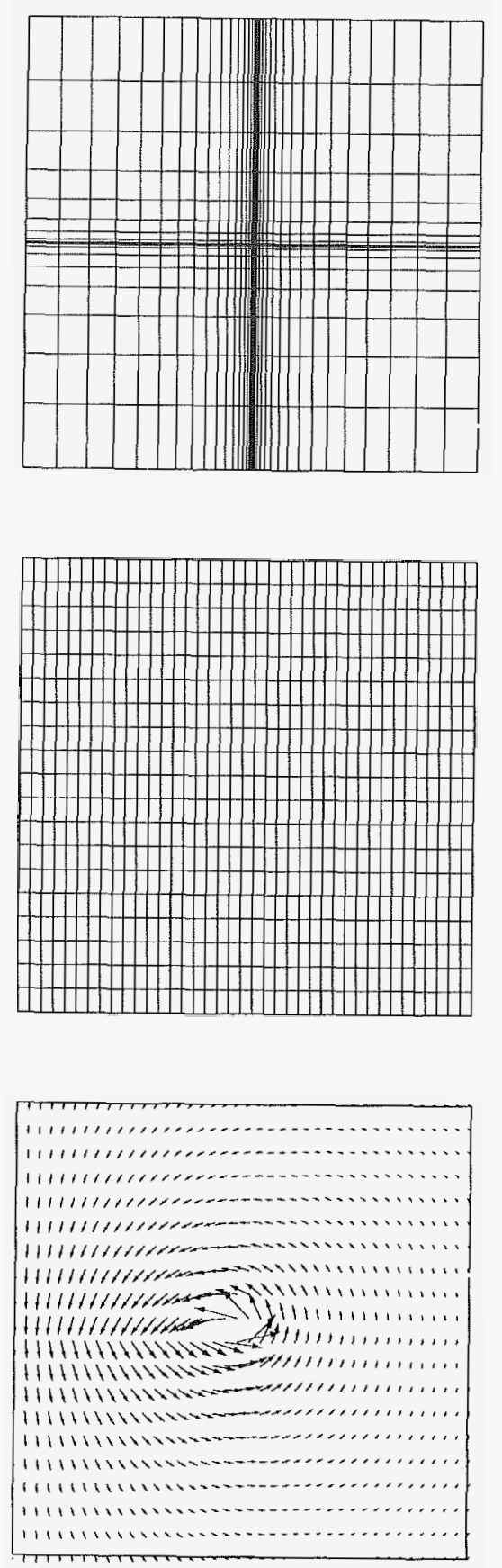

Figure 2: First test case: a) stretched grid, b) uniform grid, c) velocity vectors
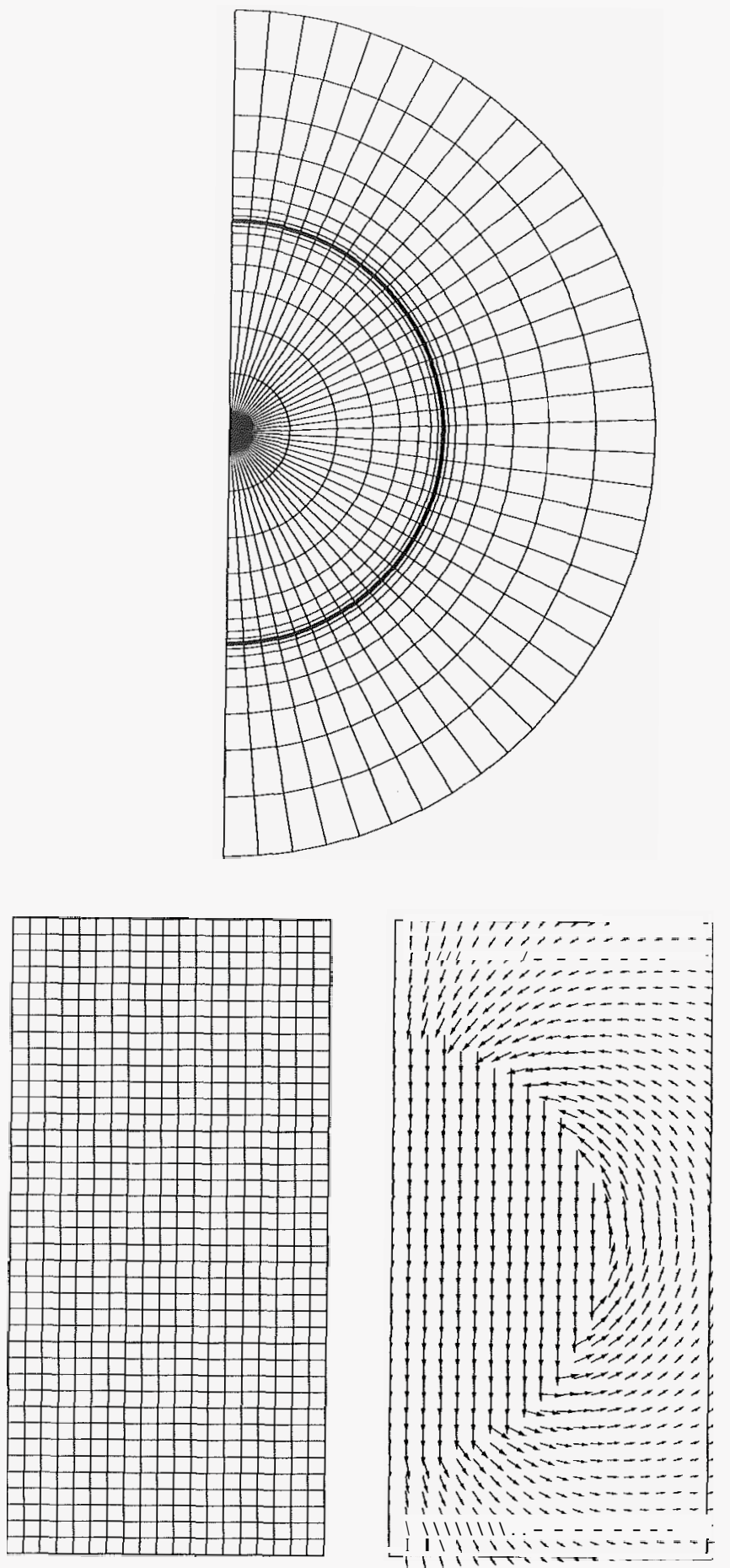

Figure 3: Second test case: a) stretched grid, b) uniform grid, c) velocity vectors 
model, based on CFD results obtained using an incompressible Navier-Stokes method [9]. As can be seen, the equivalent lifting line model predicts lift and induced drag coefficients that are within $5 \%$ of the wake survey results. The surface integration, however, badly overpredicts the total drag coefficient, probably due to a combination of turbulence modeling effects and numerical truncation errors.

This same geometry and CFD flow solution was used to evaluate the methods developed in this paper. The grid and wing geometry for the CFD calculation are shown in Fig. 4; the mesh is a single zone $81 \times 81 \times 81$ structured $\mathrm{C}-\mathrm{H}$ grid. The integrals in Equation 29 were evaluated using the CFD data from a crossflow grid plane. Figure 5 shows the resulting predictions of lift, induced drag and profile drag coefficients at various distances downstream from the wing trailing edge. The predictions are compared with tlie data from the wind tunnel test. In general, the results show that reasonable predictions of the lift and induced drag are made within three chords of the trailing edge. The profile drag coefficient is computed to be 0.022 at three chords behind the trailing edge; this is higher than the experimental value of 0.015 , but is in line with the results from the surface integration giving a total drag coefficient of 0.0413 .

Another item of interest was to verify that wake surveys predicting lift and induced drag do not need to take place across the full span and height of the wind tunnel or CFD solution. Various experimentalists have verified that one of the advantages of integrating the vorticity is that the size of the wake survey can be greatly reduced. Figure 6 shows the vorticity from the CFD solution in a crossflow plane which is 0.2 chords behind the trailing edge. The results clearly show the vast majority of the vorticity is confined to a very small area behind the wing tip and trailing edge. To further verify this, the wake survey calculations were performed again at 0.2 chords behind the trailing edge, (corresponding to Figure 6) with the vertical height of tlie integration area being restricted to values ranging from 6 chords above and below the wing surface, to within 0.1 chords of the wing. The results for lift and induced drag coefficient for these restricted integration areas are shown in Figure 7 and are compared with the available experimental data. Both coefficients are seen to be well predicted in regions as small as one chord height above and below the wing.

\section{References}

[1] A. Betz. Ein verfahren zur direkten ermittlung des profilwiderstandes. ZFM, 16:42-44, 1925.
[2] G.W. Brune and P.W. Bogataj. Induced drag at a simple wing from wake measurements. SAE Technical Paper 901934, Oct 1990.

[3] A. Chatterjee and J.M. Janus. On the use of a wake-integral method for computational drag analysis. AIAA Paper 95-0535, Jan 1995.

[4] M.B. Giles and R. Haimes. Advanced interactive visualization for CFD.

[5] H. Lamb. Hydrodynamics. Cambridge University Press, sixth edition, 1993.

[6] M.J. Lighthill. Higher Approximation, pages 345 489. OUP, 1955.

[7] R.C. Lock. Prediction of the drag of wings at subsonic speeds by viscous/inviscid interaction techniques. RAE Technical Memorandum Aero 2077, 1986.

[8] E.C. Maskell. Progress towards a method for the measurement of the components of the drag of a wing of finite span. Technical Report 72232, Royal Aircraft Establishment, 1973.

[9] D.L. Mathias, J.C. Ross, and R.M. Cummings. Wake integration to predict wing span loading from a. numerical simulation. J. Aircraft, 32(5):11651167, Sep 1995.

[10] D.J. Maull and P.W. Bearman. The measurement of the drag of bluff bodies by the wake tranverse method. J. Roy. Aeronautical Soc., 1964.

[11] Der Verdiclitungsstoss bei der stationaren Umstromung flacher Profile. ZAMM, 29:129-141, 1949.

[12] C.P. van Dam and K. Niltfetrat. Accurate prediction of drag using Euler methods. J. Aircraft, 29(3):516-519, 1992.

[13] J. van der Vooren and J. W. Slooff. CFDbased drag prediction: state-of-the-art, theory, prospects. NLR Technical Report TP 90247; also Lecture Notes for the AIAA Professional, Studies Series Course on Drag Prediction and Measurement, 1990.

[14] J.C. Wu, J.E. Hacltett, and D.E. Lilley. A generalized wake-integral approach for drag determination in three-dimensional flows. AIAA Paper 79-0279, Jan 1979. 


\begin{tabular}{|l|l|l|l|l|}
\hline & $C_{l}$ & $C_{d}$ & $C_{d p}$ & $C_{d i}$ \\
\hline experiment: wake survey & 0.5657 & 0.0321 & 0.0151 & 0.0170 \\
experiment: balance data & 0.5723 & 0.0319 & & \\
CFD: lifting line model & 0.551 & & & 0.016 \\
CFD: surface integration & 0.560 & 0.0413 & & \\
\hline
\end{tabular}

Table 1: Experimental and CFD lift and drag coefficients for Brume and Bogataj test case

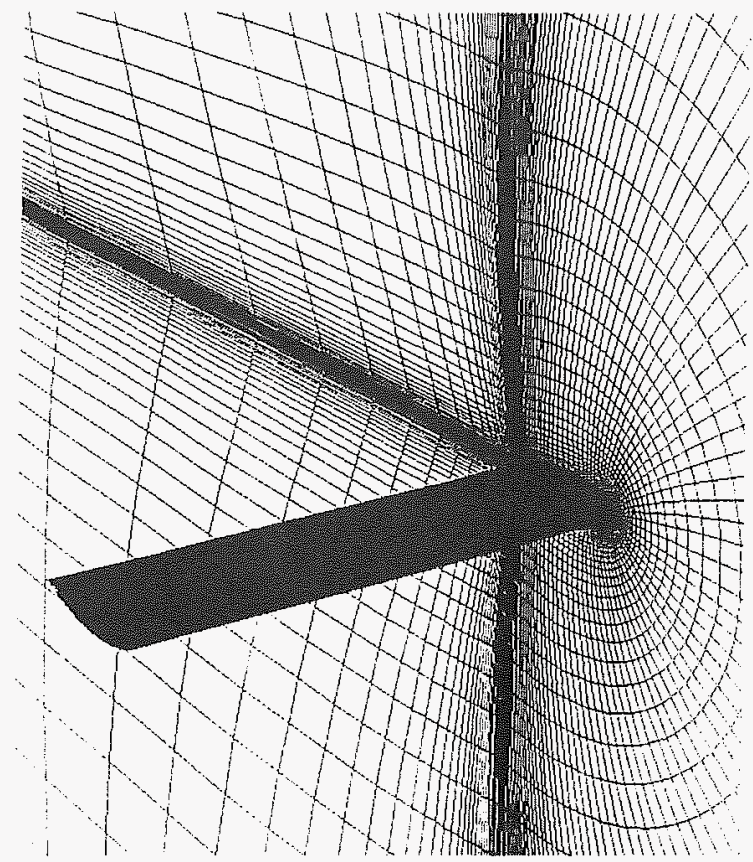

Figure 4: NACA 0016 wing surface and C-H grid $(\mathbf{8 1} \times 81 \times 81)$
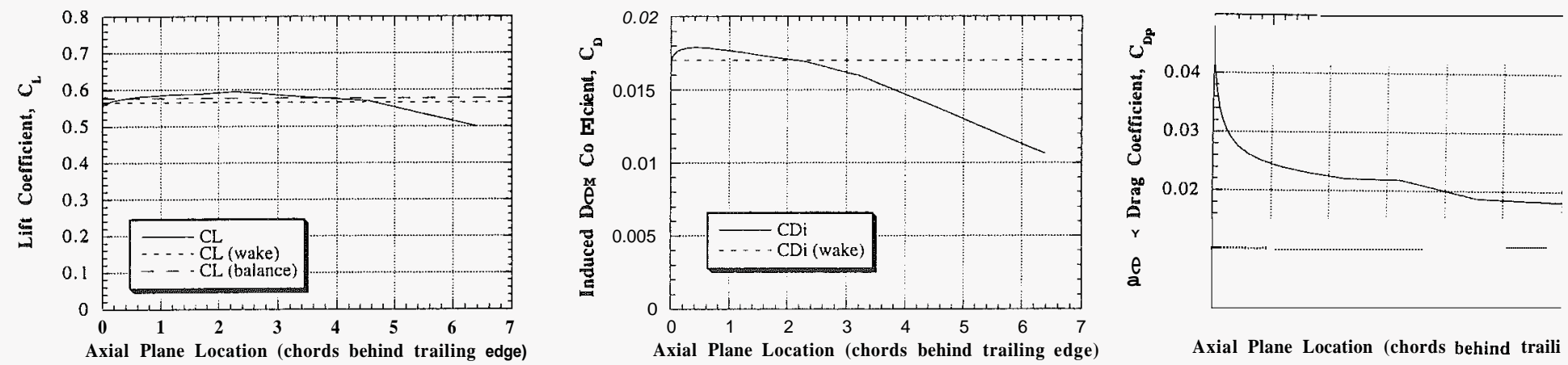

Axial Plane Location (chords behind traili

Figure 5: Lift, induced drag, and profile drag coefficients at various axial plane locations 


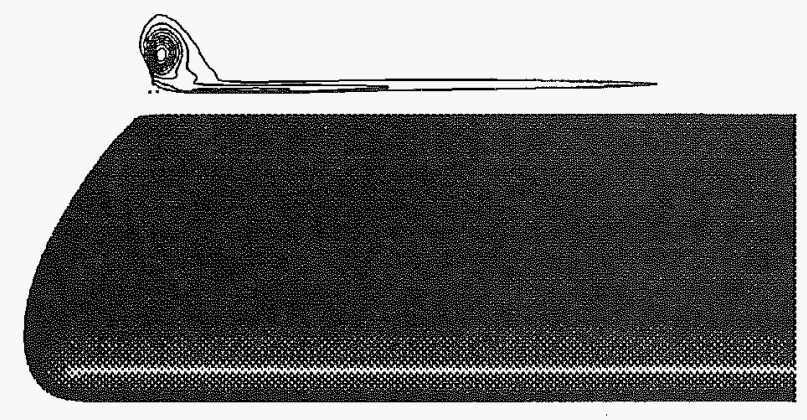

Figure 6: Vorticity in cross-flow plane at $x / c=0.2$ chords behind trailing edge
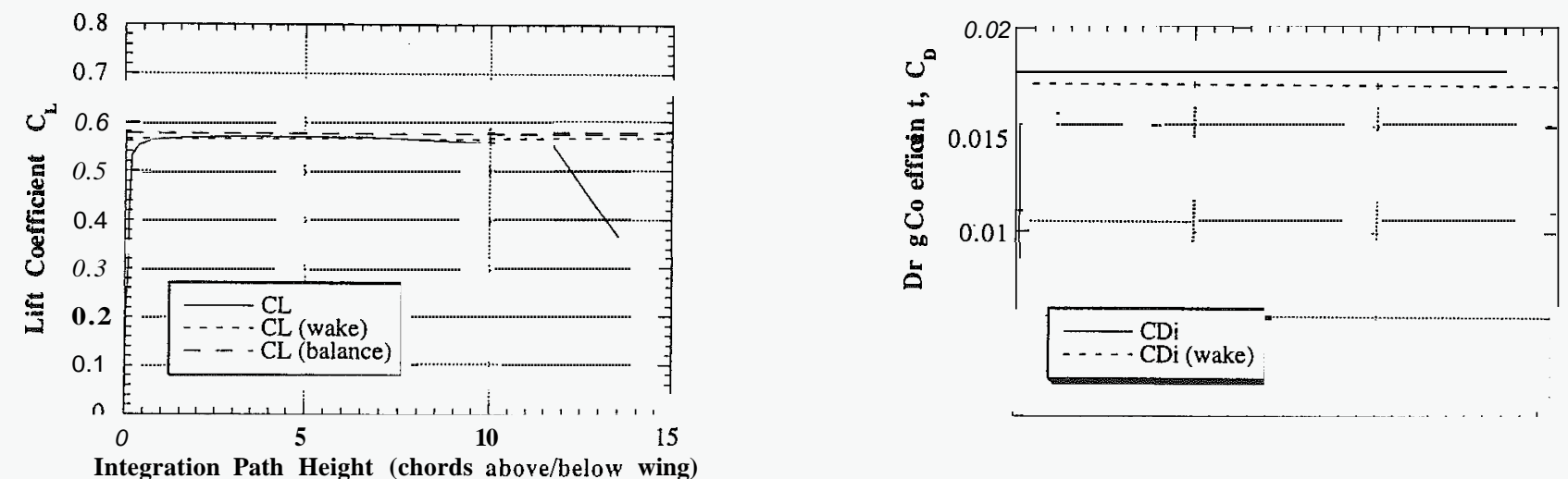

Integration Path Height (chords above/below wing)

Figure 7: Lift and induced drag coefficients for varying integration heights 